\title{
Внутрицентровые излучательные переходы на примесных центрах железа в селениде цинка
}

\author{
(C) В.В. Ушаков, Д.Ф. Аминев, В.С. Кривобок \\ Физический институт им. П.Н. Лебедева Российской академии наук, \\ 119991 Москва, Россия \\ E-mail: ushakovvv@lebedev.ru
}

Поступила в Редакцию 19 ноября 2020 г.

В окончательной редакции 10 декабря 2020 г.

Принята к публикации 10 декабря 2020 г.

\begin{abstract}
В области 0.6-1.8 мкм исследован спектральный состав люминесценции образцов ZnSe, легированных примесью железа методом высокотемпературной термодиффузии в атмосфере Zn или Ar. B спектрах люминесценции образцов наблюдались линии при 980 и 1400 нм, связанные с внутрицентровыми излучательными переходами в центрах $\mathrm{Fe}^{2+}$. По данным спектрального сканирования, интенсивности обеих линий коррелировали по всей поверхности образца, что свидетельствует о связи обоих излучательных переходов с одним и тем же центром. Идентификация излучательных переходов проведена по диаграмме Танабе-Сугано с параметрами, адаптированными к центрам $\mathrm{ZnSe}: \mathrm{Fe}^{2+}\left(d^{6}\right)$. Результаты свидетельствуют о том, что линии при 980 и 1400 нм обусловлены электронными переходами ${ }^{3} T_{1}\left({ }^{3} H\right)-{ }^{5} E\left({ }^{5} D\right)$ и ${ }^{5} T_{2}\left({ }^{5} D\right)$ соответственно.
\end{abstract}

Ключевые слова: люминесценция, внутрицентровые переходы, $\mathrm{ZnSe}: \mathrm{Fe}$.

DOI: 10.21883/FTP.2021.04.50729.9556

\section{1. Введение}

В настоящее время существует все возрастающая потребность в компактных, работающих при комнатной температуре лазерных устройствах среднего инфракрасного диапазона. Спектр применения таких систем чрезвычайно широк: газовые анализаторы, медицина, оптическая связь, различные области военного/двойного назначения и др.

В этой связи перспективными в качестве активного материала для перестраиваемых лазеров являются кристаллические соединения II-VI с локальными центрами переходных элементов группы железа. Для достижения наибольшей эффективности в работе прибора необходимо иметь разработанную технологию получения легированных кристаллов, а также изучить энергетическую структуру и свойства электронных переходов для активного элемента. Последнее необходимо для выбора наиболее эффективной схемы передачи энергии первоначального возбуждения непосредственно к „лазерному“ переходу.

В данной работе мы рассмотрели часть обозначенных вопросов применительно к системе $\mathrm{ZnSe}: \mathrm{Fe}$.

\section{2. Получение образцов и методика эксперимента}

Исходные оразцы $\mathrm{ZnSe}$ прямоугольной формы с размерами $10 \times 3 \times 2$ мм были вырезаны из пластины крупноблочного материала с размерами зерен 200-300 мкм, полученного методом химического осаждения из газовой фазы и прошедшего тщательную оптическую полировку. Легирование железом проводилось методом термодиффузии, при этом на одну из боковых сторон $10 \times 2$ мм методом электронно-лучевого испарения была нанесена металлическая пленка $\mathrm{Fe}$ толщиной 1 мкм, которая после отжига в атмосферах $\mathrm{Zn}$ или $\mathrm{Ar}$ при температуре $1100^{\circ} \mathrm{C}$ в течение 27 ч была удалена. Процедура приготовления образцов подробно описана в [1]. Распределение железа в плоскости измерений (перпендикулярно плоскости легирования) было известно по измерениям оптического пропускания на длине волны 2.8 мкм, соответствующей, согласно [2], одной из полос поглощения центров $\mathrm{ZnSe}: \mathrm{Fe}^{2+}$. Максимальная концентрация легирующей примеси (в оптически активном состоянии) составляла $\sim 10^{19} \mathrm{~cm}^{-3}[1]$, оптимальную величину для лазерных устройств на $\mathrm{ZnSe}: \mathrm{Fe}$. Кроме того, при использованной схеме легирования имелась возможность проследить взаимодействие атомов железа с фоновыми примесями или дефектами при различной концентрации легирующей примеси.

Люминесцентные измерения выполнялись на автоматизированном микрофотолюминесцентном сканере с минимальным шагом 5 мкм и возможностью спектрального анализа излучения в выбранных точках на поверхности образцов и их люминесцентного картирования на заданной длине волны. Этот метод особенно информативен при исследовании неоднородных люминесцирующих систем. Измерения проводились при температурах 90-300 К. Мощность возбуждающего излучения TEM $_{00}$ диодного лазера DTL-379 (473 нм) на поверхности образцов составляла 2-3 мВт при диаметре пятна 15 мкм. Спектральный анализ люминесценции в области 0.6-1.8 мкм осуществлялся с помощью монохроматора МДР-12 с дифракционной решеткой 600 штр/мм (обратная линейная дисперсия 4.8 нм/мм) и охлаждаемой азотом детекторной системой на основе 
Ge- $p-i-n$-диода (North Coast) с синхронным детектированием сигнала.

\section{3. Результаты и их обсуждение}

\section{1. Спектры люминесценции}

В лазерных системах на основе кристаллической матрицы $\mathrm{ZnSe}$ участвуют изолированные центры $\mathrm{Fe}$, располагающиеся в катионных узлах решетки с зарядовым состоянием $\mathrm{Fe}^{2+}$, нейтральные по отношению к решетке. По данным электронного парамагнитного резонанса (ЭПР), в образцах $\mathrm{ZnSe}: \mathrm{Fe}$ присутствуют также центры $\mathrm{Fe}^{3+}$ [3-6], причем по результатам [5] центры 2+ и 3+ могут присутствовать в одном кристалле одновременно. Результаты данной работы по методу определения (см. разд. 2) относятся к центрам $\mathrm{ZnSe}: \mathrm{Fe}^{2+}$.

На рис. 1 приведен спектр люминесценции образца $\mathrm{ZnSe}: \mathrm{Fe}$ при температуре $90 \mathrm{~K}$. Минимум в центре более слабой линии 1400 нм связан с поглощением излучения парами атмосферной воды (cp. с [5]). Концентрация $\mathrm{Fe}^{2+}$ в точке измерений $10^{18} \mathrm{~cm}^{-3}$. Интенсивная линия при 980 нм с полушириной $\sim 90$ нм в $\mathrm{ZnSe}$ (и в близком по свойствам $\mathrm{ZnS}$ ) известна еще по публикациям 1980-х годов, где ее связывали с внутрицентровыми излучательными переходами в центрах $\mathrm{Fe}_{\mathrm{Zn}}^{2+}$ между уровнями с различными значениями спина ${ }^{3} T_{1}-{ }^{5} E$ [7]. Линия при 1400 нм наблюдалась в немногих работах (см., например, $[4,7])$, где предложительно была отнесена к переходам ${ }^{3} T_{1}-{ }^{5} T_{2}$ на тех же центрах. Необходимо иметь в виду, что для соединений II-VI в оптических спектрах внутрицентровых электронных переходов примесных центров элементов группы железа слабые бесфононные линии наблюдаются, как правило, лишь до температур $\sim 20 \mathrm{~K}$, так что при азотных температурах в спектрах остаются лишь интенсивные полосы фононных крыльев.

Для образцов, отожженных в атмосфере Ar, наблюдался точно такой же спектр, но со значительно меньшей

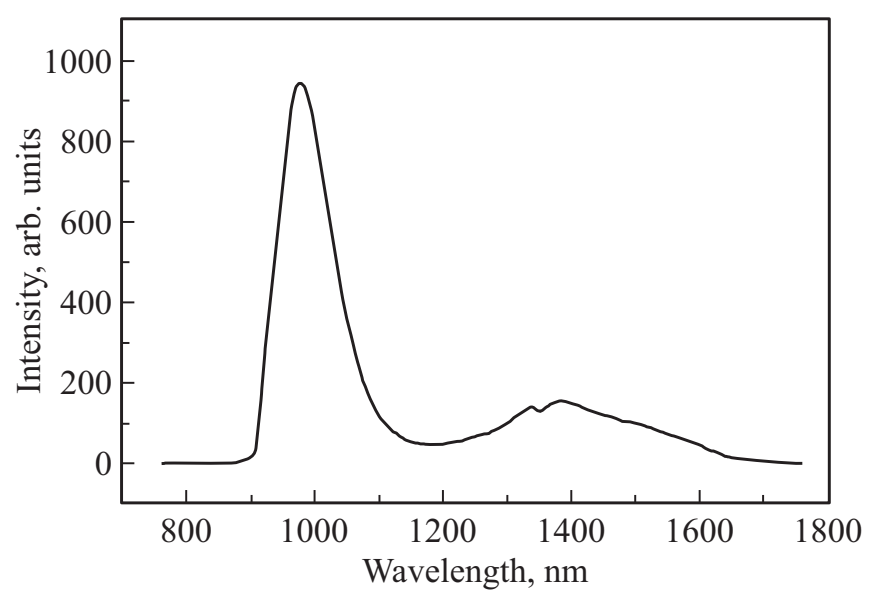

Рис. 1. Спектр ZnTe: Fe при $90 \mathrm{~K}$.

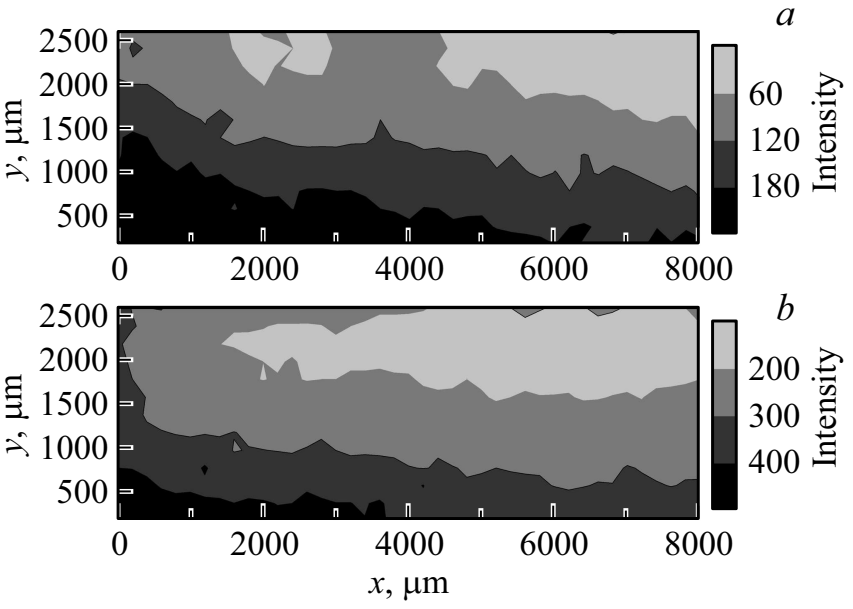

Рис. 2. Картограммы образца $\mathrm{ZnTe}: \mathrm{Fe}$ в спектральных линиях $980(a)$ и 1400 нм $(b)$ при $90 \mathrm{~K}$ в координатах $x, y$.

интенсивностью линий. Это, очевидно, связано с тем, что атомы Zn из атмосферы, в которой проходил отжиг, эффективно компенсируют потерю „летучей“ компоненты матрицы в процессе отжига.

Для технологического контроля исследования термической диффузии легирующей примеси, а также для исследования взаимодействия атомов железа с фоновыми примесями/дефектами при различной концентрации легирующей примеси было проведено картирование всего образца на длинах волн, соответствующих максимумам наблюдавшихся в спектре (рис. 1) полос. Эти результаты приведены на рис. 2 для $1400(a)$ и 980 нм $(b)$. Шаг сканирования - 100 мкм. Интенсивность люминесценции кодирована шкалой, расположенной справа от соответствующей картограммы. В обоих случаях максимальная интенсивность люминесценции наблюдалась в областях, примыкавших к торцу, где первоначально была нанесена пленка металлического $\mathrm{Fe}$, и затем постепенно уменьшалась к противоположной стороне образца. По всему образцу характер этой зависимости для обеих полос принципиально не различается. Это свидетельствует, в частности, о том, что оба излучательных перехода, рассмотренных в этой работе, происходят на одном и том же центре. Наглядной иллюстрацией сказанному является корреляция зависимостей интенсивностей обеих полос по линейному срезу двумерных картограмм люминесценции по координате $x=0.8$ мм (рис. 3). Особенность зависимостей для обеих полос состоит в том, что значения их интенсивностей на крае образца отлична от 0 . Это можно объяснить либо ускоренной диффузией железа в исследованных образцах (большая концентрация термодефектов - отжиг при $\left.1100^{\circ} \mathrm{C}\right)$, либо заметной концентрацией фонового железа.

\section{2. Идентификация электронных переходов}

Встроившиеся в кристаллическую решетку ZnSe атомы Fе имеют внешнюю („оптическую“) электронную 


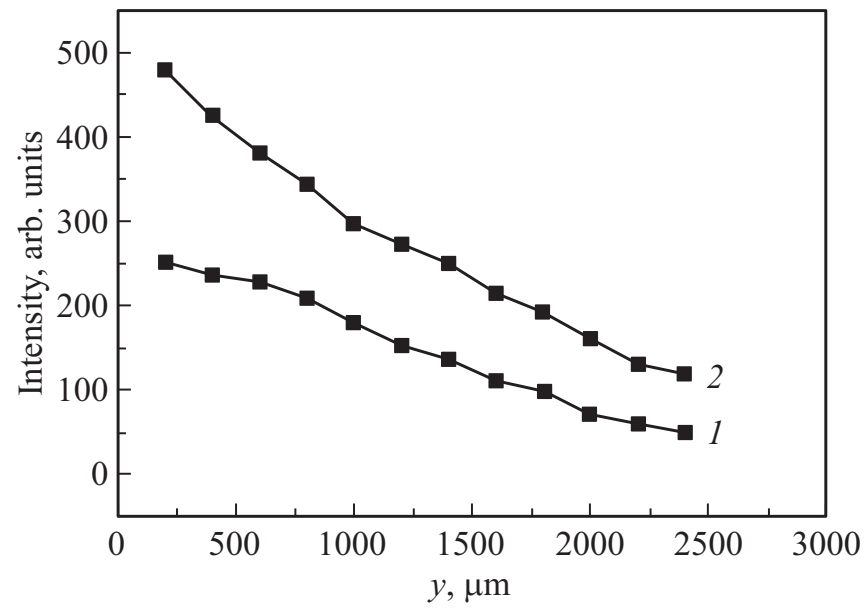

Рис. 3. Зависимости интенсивностей спектральных линий 980 (1) и 1400 нм (2) от координаты у по срезу картограмм люминесценции при $x=0.8$ мм (см. текст).

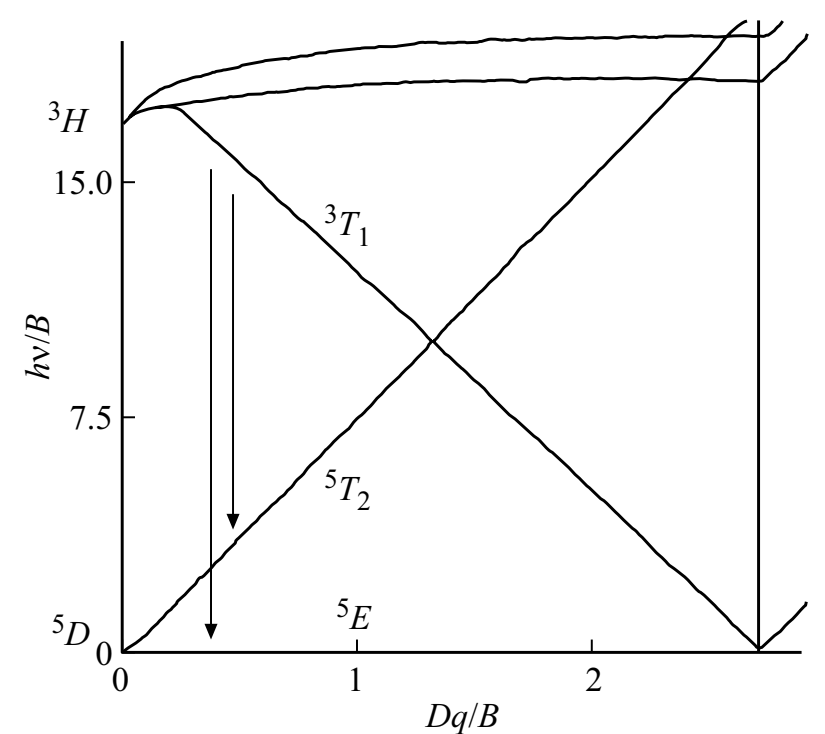

Рис. 4. Идентификация электронных переходов, связанных с линиями люминесценции 980 и 1400 нм, по диаграмме ТанабеСугано (см. текст).

конфигурацию $d^{6}$ (состояние $\mathrm{Fe}^{2+}$ ), находящуюся в тетраэдрическом кристаллическом поле первой $\left(T_{d}\right)$ координационной сферы. Энергетическим свойствам $d$-электронных систем в кристаллах посвящена монография [8]. Применительно к центрам $\mathrm{Fe}^{2+}\left(d^{6}\right)$ атомный терм основного состояния ${ }^{5} D$ (25-кратно орбитально вырожденный) в поле симметрии $T_{d}$ расщепляется на дуплет ${ }^{5} E$ и триплет ${ }^{5} T_{2}$ (соответственно 10 - и 15 -кратно вырожденные). Дальнейшие расщепления уровней в результате спин-орбитального взаимодействия (см., например, [7]), равно как и эффектов ян-теллеровского взаимодействия [4], для центров $\mathrm{ZnSe}: \mathrm{Fe}$ в матрицах II-VI имеют порядок нескольких мэВ. Температуры измерений в данной работе были велики $(\geq 90 \mathrm{~K})$, так что в экспериментальных спектрах эти детали не могли проявляться. Поэтому для интерпретации спектров при $90 \mathrm{~K}$ достаточно TS-диаграммы $d^{6}$-электронной системы в $T_{d}$-кристаллическом поле, учитывающей лишь кристаллическое поле и межэлектронное взаимодействие [8].

На рис. 4 представлена (частично, применительно к данной работе) TS-диаграмма с зависимостью энергии атомных термов от величины кристаллического поля $D q$ (все в единицах параметра межэлектронного взаимодействия Рака́ $B$ ). Диаграмма адаптирована к случаю $\mathrm{ZnSe}: \mathrm{Fe}$, а именно принято: $\Delta=10 D q=3000 \mathrm{~cm}^{-1}$ [9] и $B=600 \mathrm{~cm}^{-1}$ (с учетом данных [7] для $\mathrm{ZnS}$ ). Это дает значение $D q / B=0.5$, что связывает оптические полосы экспериментального спектра рис. 1 с электронными переходами ${ }^{3} T_{1}\left({ }^{3} H\right)-{ }^{5} E\left({ }^{5} D\right)$ (энергия $10200 \mathrm{~cm}^{-1}$, длина волны 980 нм) и ${ }^{3} T_{1}\left({ }^{3} H\right)-{ }^{5} T_{2}(5 D)\left(7140 \mathrm{~cm}^{-1}, 1400 \mathrm{нм}\right)$. На TS-диаграмме рис. 4 указанные переходы отмечены стрелками. Поскольку оба перехода происходят с изменением спина, их интенсивность на 3 порядка меньше используемого в ,лазерной“ технике ${ }^{5} T_{2}\left({ }^{5} D\right)-{ }^{5} E\left({ }^{5} D\right)$.

\section{3. Схемы возбуждения люминесценции}

Зарядовое состояние переходных элементов в кристаллах II-VI и процессы, приводящие к его изменению, рассмотрены в [6]. В лазерных системах на основе кристаллических матриц $\mathrm{ZnSe}$ участвуют изолированные центры $\mathrm{Fe}$, располагающиеся в катионных узлах решетки с зарядовым состоянием $\mathrm{Fe}^{2+}$, нейтральные по отношению к решетке. По данным ЭПР, в образцах $\mathrm{ZnSe}: \mathrm{Fe}$ присутствуют также центры $\mathrm{Fe}^{3+}[4,5]$, причем, согласно $[5,10]$, центры $2+$ и $3+$ могут присутствовать в одном кристалле одновременно. Центры $\mathrm{Fe}^{3+}$ нередко участвуют в процессах трансформации энергии внешнего возбуждения к энергетическим уровням ,лазерных“ центров $\mathrm{Fe}^{2+}$. В кристаллах II-VI межзонное возбуждение не приводит к внутрицентровой люминесценции железа. Для этого необходимо подзонное возбуждение. Для $\mathrm{ZnSe}: \mathrm{Fe}^{2+}$ максимальная интенсивность достигается при использовании лазеров на 525 нм при коэффициенте поглощения $4 \mathrm{~cm}^{-1}$. В данной работе 473 нм. При таких энергиях возможно поглощение возбуждения на каком-то объекте (примесном центре) с последующей передачей энергии на „лазерные“ центры $\mathrm{Fe}^{2+}$. Например, для $\mathrm{ZnSe}: \mathrm{Fe}$ она впервые предложена в [7] и в общем виде может быть записана как $\mathrm{Fe}^{3+}+e \rightarrow \mathrm{Fe}^{2+}\left({ }^{3} T_{1}\right)-980 \mathrm{Hм}-\mathrm{Fe}^{2+}\left({ }^{5} E\right)$ (захват электрона с донора центром $\mathrm{Fe}^{3+}$ ).

\section{4. Заключение}

По данным люминесценции, для использованной технологии высокотемпературный отжиг в атмосфере $\mathrm{Zn}$ имеет несомненные преимущества против аналогичной процедуры в атмосфере инертного Ar. Это, очевидно, связано с тем, что атомы $\mathrm{Zn}$ из атмосферы, в которой 
проходил отжиг, эффективно компенсируют потерю „летучей“ компоненты матрицы.

Спектральное картирование образцов отражает детали использованной технологии. Интенсивности спектральных полос при 980 и 1440 нм по всей поверхности образцов, очевидно, коррелируют.

Идетификация излучательных переходов для центров $\mathrm{ZnSe}: \mathrm{Fe}^{2+}\left(d^{6}\right)$ в спектральной области 0.6-1.8 мкм проведена по диаграмме Танабе-Сугано, с параметрами, адаптированными к рассматриваемому случаю. Результаты с очевидностью свидетельствуют о том, что полоса 980 нм связана с излучательным переходом ${ }^{3} T_{1}\left({ }^{3} H\right)-{ }^{5} E\left({ }^{5} D\right)$, a $1440 \mathrm{Hм}-\mathrm{c}^{3} T_{1}\left({ }^{3} H\right)-{ }^{5} T_{2}\left({ }^{5} D\right)$.

\section{Финансирование работы}

Работа выполнена при финансовой поддержке Российского научного фонда (грант 19-79-30086).

\section{Конфликт интересов}

Авторы заявляют, что у них нет конфликта интересов.

\section{Список литературы}

[1] А.А. Пручкина, Д.Ф. Аминев, В.В. Ушаков, С.И. Сенцов, А.А. Гладилин, В.С. Кривобок, Е.Е. Онищенко, В.П. Калинушкин. КСФ, 46 (7), 42 (2019). [Bull. Lebedev Physics Institute, 46, 238 (2019)].

[2] J. Peppers, V. Fedorov, S. Mirov. Opt. Express, 23, 4406 (2015).

[3] Physics and Chemistry of $A^{I I} B^{V I}$ Compounds, ed. by M. Aven, J.S. Prener (Amsterdam, North-Holland, 1967).

[4] K.P. O’Donnel, K.M. Lee, G.D. Watkins. J. Phys. C, 16, L723 (1983).

[5] L.L. Kulik, R. Laiho, A.V. Lashkul, E. Lahderanta, D.D. Negeoglo, N.D. Negeoglo, I.V. Radevuci, A.V. Simmel, V.P. Sirkeli, K.D. Suskevich. Physica B, 405, 4330 (2010).

[6] J. Kreissl, H.-J. Schulz. J. Cryst. Growth, 161, 239 (1996).

[7] M. Skowronski, Z. Liro. J. Luminesc., 24/25, 253 (1981).

[8] S. Sugano, Y. Tanabe, H. Kamimura. Multiplets of TransitionMetal Ions in Crystals (N.Y., Academic Press, 1970).

[9] J.W. Evans, T.R. Harris, B.R. Reddy, K..L. Schepler, P.A. Berry. J. Luminesc., 188, 541 (2017).

[10] V.V. Fedorov, S.B. Mirov, A. Gallian, D.V. Badikov, M.P. Frolov, Yu.V. Korostelin, V.I. Kozlovsky, A.I. Landman, Yu.P. Podmarkov, V.A. Akimov, A.A. Voronov. IEEE J. Quant. Electron., 42, 907 (2006).

Редактор Л.В. Шаронова

\section{Intracenter radiative transitions at ZnSe: Fe impurity centers in the near infrared region of the spectrum}

\author{
V.V. Ushakov, D.F. Aminev, V.S. Krivobok \\ Lebedev Physical Itstitute, \\ Russian Academy of Sciences, \\ 119991 Moscow, Russia
}

Abstract In the range $0.6-1.8 \mu \mathrm{m}$, the luminescence spectral composition of $\mathrm{ZnSe}$ samples doped with an iron impurity by high-temperature thermal diffusion in a $\mathrm{Zn}$ or $\mathrm{Ar}$ atmosphere was studied. In the luminescence spectra of the samples, 980 and $1400 \mathrm{~nm}$ lines, associated with intracenter radiative transitions at $\mathrm{Fe}^{2+}$ centers, were observed. According to the data of spectral scanning, the intensities of both lines were correlated over the entire surface of the sample, which indicates that both radiative transitions occur at the same center. The radiative transitions were identified using the Tanabe-Sugano diagram with parameters adapted to $\mathrm{ZnSe}: \mathrm{Fe}^{2+}$ centers $\left(d^{6}\right)$. The results indicate that the 980 and $1400 \mathrm{~nm}$ lines are associated with the ${ }^{3} T_{1}\left({ }^{3} H\right)-{ }^{5} E\left({ }^{5} D\right)$ and ${ }^{5} T_{2}\left({ }^{5} D\right)$ electron transitions, respectively. 